\title{
Pulmonary interstitial fibrosis associated with alveolar proteinosis
}

\author{
HW CLAGUE, AC WALLACE, WKC MORGAN \\ From the Departments of Medicine and Pathology, University Hospital, University of Western Ontario, \\ London, Ontario, Canada
}

\begin{abstract}
Alveolar proteinosis is characterised by the presence of an amorphous lipoproteinaceous material that fills the alveolar space and stains positively by the periodic acid Schiff (PAS) method.' Alveolar walls are usually normal or only slightly thickened by cellular infiltrate, and interstitial fibrosis is unusual. We report a patient in whom severe interstitial fibrosis occurred early in the course of the disease and led to a delay in correct diagnosis and to inappropriate treatment with corticosteroids.
\end{abstract}

\section{Case report}

A 54 year old man who worked as a forklift truck driver presented with a two month history of effort dyspnoea, recurrent painful pleurisy, and fever with cough and sputum production. Physical examination revealed nothing abnormal but a chest radiograph showed basal interstitial nodular shadowing. A needle lung biopsy specimen ${ }^{2}$ (fig 1) from the left lower lobe was reported as showing interstitial fibrosis with considerable numbers of both interstitial and intra-alveolar macrophages. Despite treatment with oral prednisone (15 $\mathrm{mg}$ daily) the patient's dyspnoea worsened and the chest radiograph showed the development of extensive perihilar ground glass shadowing. Occasional paninspiratory crackles were audible at the lung bases. Pulmonary function testing showed a restrictive ventilatory defect (FVC $70 \%$ predicted; FEV $183 \%$ predicted; $\mathrm{FEV}_{1} / \mathrm{FVC}$ ratio $88 \%$ ), with a reduced gas transfer factor (29\% predicted).

Six months later there was symptomatic improvement and significant radiological clearing. The clinical course, the lack of response to steroids, and the more recent radiographic changes led to a clinical suspicion of alveolar proteinosis. A further needle lung biopsy ${ }^{2}$ of the left lower lobe was therefore performed, but microscopic appearances confirmed interstitial fibrosis with intra-alveolar organisation and an accumulation of foamy macrophages. Two months later the patient presented with an acute febrile illness, haemoptysis, and an infective arthritis of the left ankle joint. The chest radiograph showed a dense right upper lobe infiltrate suggestive of tuberculosis. During a search of sputum and joint aspirate for Mycobacterium tuberculosis a truant rhodamine $\operatorname{stain}^{3}$ demonstrated the presence of large numbers of pus cells and Gram positive fluorescent filamentous rods, which on culture were identified as Nocardia asteroides. Administration of amox-

Address for reprint requests: Dr HW Clague, Chest Unit, Fazakerley Hospital, Liverpool L9 7AL.

Accepted 11 March 1983

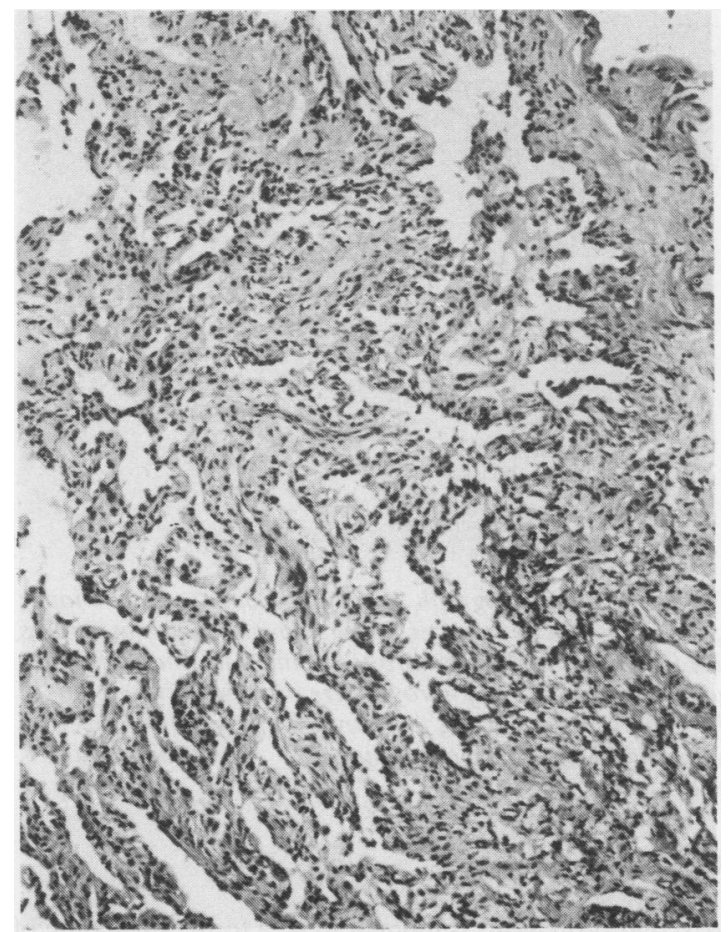

Fig 1 Appearances of first (needle) lung biopsy from left lobe (lower) showing alveolar hyperplasia with dense interstitial fibrosis but no alveolar exudate. (Haematoxylin and eosin, $\times$ 185.)

ycillin trihydrate $0.5 \mathrm{~g}$ four hourly for four weeks and sulphafurazole $2 \mathrm{~g}$ four hourly for eight weeks, with repeated joint aspiration, led to complete resolution of both the pneumonia and the infective arthritis.

Twelve months later the patient's dyspnoea had again worsened and fine end inspiratory crackles became audible at the left apex. At this stage an open lung biopsy of the left upper and left lower lobe was performed and on this occasion the main microscopic feature was the presence in both biopsy specimens of an amorphous intra-aveolar accumulation of PAS positive proteinaceous material (fig 2). In places the alveolar walls were disrupted with evidence of only slight thickening and fibrosis; but in other areas pronounced fibrosis of the interstitial tissue was evident, with replacement of alveolar walls by dense collagen. 


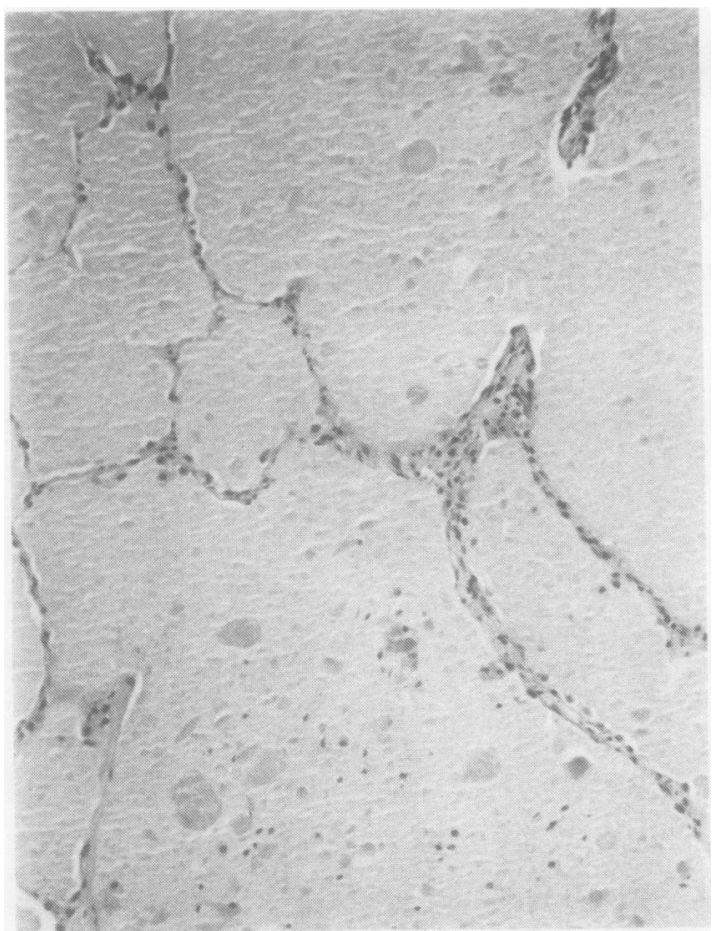

Fig 2 Photomicrograph of third (open) lung biopsy specimen from left upper lobe, showing alveolar spaces filled with eosinophilic amorphous material typical of alveolar proteinosis; foci of fibrosis were present elsewhere but in this area alveolar walls are only slightly thickened with hyperplasia of the alveolar lining cells. (Trichrome stain, $\times$ 185.)

\section{Discussion}

Alveolar proteinosis is thought to be a form of response of the lung to various noxious agents. Inhalation of high concentrations of fine silica dust has been especially implicated, although in most cases the cause is not apparent.' Experimental work and electronmicroscopic examination point to hyperplasia of the type 2 granular pneumocyte. as the initial event, with stimulation of these cells to produce large quantities of an amorphous lipoproteinaceous material, ${ }^{4}$ which is structurally similar to surfactant although lacking its surface properties. The material usually stains homogeneously but may have a distinctly granular appearance with clear acicular clefts and numerous laminated bodies. $^{5}$ The lipoprotein produced fills or distends the alveolar space and overwhelms the mechanisms for lung clearance, leading to an impairment of gas exchange and even death from respiratory failure. As in the present case, the alveolar septa are sometimes disrupted by overdistension to form large lakes of eosinophilic staining material. ${ }^{6}$
Pulmonary fibrosis is uncommon even in fatal cases and the respiratory bronchioles and alveolar walls are usually of normal thickness, but may be slightly thickened owing to a mainly lymphocytic infiltration. ${ }^{1}$ This contrasts with the findings in our patient, in whom there was evidence of severe interstitial fibrosis early in the course of the disease. The results of our first biopsy misled us into using corticosteroids, which are of no value in alveolar proteinosis and may even prove harmful by predisposing to opportunist infection. The lung macrophage in alveolar proteinosis has reduced phagocytic potential ${ }^{7}$ and, perhaps because of this, patients are already predisposed to opportunist infection. An association with nocardiosis has been well described. ${ }^{9}$

Focal interstitial fibrosis of varying degree but of questionable significance has been reported previously. ${ }^{589}$ The presence of patchy mild interstitial fibrosis has in some cases been attributed to the presence of opportunist infections, but this cannot be true of our patient as the development of fibrosis clearly predated infection with nocardia. In this case interstitial fibrosis appears to represent a late response to alveolar proteinosis. Hudson and associates ${ }^{10}$ have reported a case of a patient who died with severe pulmonary fibrosis some 13 years after an initial diagnosis of alveolar proteinosis.

Open lung biopsy may have given a more representative sample earlier on in our patient. Good results have however been claimed for diffuse lung diseases with the techniques that we used. ${ }^{2}$

As the aetiological agent was not identified in the case that we report, it is possible that the offending agent was coincidentally fibrogenic. Nevertheless, our report demonstrates that the presence of severe interstitial fibrosis does not preclude a diagnosis of alveolar proteinosis.

\section{References}

' Spencer, H. Pathology of the lung. 3rd ed. Vol 2. Oxford: Pergamon Press, 1978:669-73.

${ }^{2}$ Newhouse MT. Suction excision biopsy for diffuse pulmonary disease. Chest 1973;63:707-12.

${ }^{3}$ Lannette EH, ed. Truant auramine-rhodamine stain for mycobacteria. Manual of clinical microbiology. 3rd ed Washington: American Society of Microbiology, 1980:1022.

${ }^{4}$ Heppleston AG, Young AE. Alveolar lipoproteinosis: an ultrastructural comparison of the experimental and human forms. $J$ Pathol 1972;107:107-17.

${ }^{5}$ Rosen SH, Castleman B, Liebow AA. Pulmonary alveolar proteinosis. N Engl J Med 1958;258:1123-42.

- Mendenhall E, Solu S, Easom HF. Pulmonary alveolar proteinosis. Am Rev Respir Dis 1961;84:876-80.

${ }^{7}$ Golde DW, Territo M, Finley TN, Cline MJ. Defective lung macrophages in pulmonary alveolar proteinosis. Ann Intern Med 1976;85:304-9.

${ }^{8}$ Fraimow W, Cathcart RT, Kirshner JJ, Taylor RC. Pulmonary $\Omega$ alveolar proteinosis: a correlation of pathological and $N$ physiological indings in a patient followed up with serial biopsies of the lung. Am J Med 1960;28:458-67.

- Davidson JM, Macleod WM. Pulmonary alveolar proteinosis. $\mathrm{Br}$ $J$ Dis Chest 1969;63:13-28.

${ }^{10}$ Hudson AR, Halprin GM, Miller JA, Kilburn KH. Pulmonary interstitial fibrosis following alveolar proteinosis. Chest \& 1974;65:700-2. 